cardiovascular disease to reduce metabolic risk. A multidisciplinary approach involving hepatologists and diabetologists alongside allied health professionals providing structured lifestyle advice is advocated. Objective evaluations of this approach are limited.

Methods We undertook a retrospective study to determine the impact of a large, tertiary centre, multidisciplinary metabolic hepatology clinic. Detailed health parameters and surrogate markers for liver and cardio-metabolic disease were evaluated and a health economic analysis was performed.

Results 165 patients with NAFLD without hepatic co-morbidity and excluding those undergoing bariatric surgery, and who attended $\geq 2$ times between 2014-17, were followed from referral until latest review. Median follow-up was 13 months (2-34). At baseline, 29\% had cirrhosis and 59\% had T2DM. At follow-up, median liver stiffness, measured using transient elastography, decreased by $1.3 \mathrm{kPa}(14 \%, \mathrm{p}=0.0097)$ and was associated with significant improvement in alanine aminotransferase (ALT: $-11 \mathrm{IU} / 1,21 \%, \mathrm{p}<0.0001)$. Median weight fell by $3.3 \mathrm{~kg}(3.4 \%, \mathrm{p}=0.0005)$ as did total cholesterol $(0.7 \mathrm{mmol} / \mathrm{L}$; $14 \%, \mathrm{p}=0.0023)$. Median HbA1c also fell $(1.5 \mathrm{mmol} / \mathrm{mol}$, $3.1 \%, \mathrm{p}=0.0045)$. Reduction was most marked in those with poorly controlled T2DM (HbA1c $>58 \mathrm{mmol} / \mathrm{mol}$ at baseline: $14 \mathrm{mmol} / \mathrm{mol}, 18 \%, \mathrm{p}<0.0001)$. These improvements Resulted in a $6.4 \%$ reduction in 10 year cardiovascular risk (QRISK3, aged-match, $\mathrm{p}=0.0085$ ).

Preliminary economic analysis of our approach using the UKPDS Outcomes Model in patients with poorly controlled diabetes indicated improvement in quality adjusted life expectancy alongside a reduction in costs of complications if health improvements were maintained. Importantly, preliminary estimates appeared to be below the cost-per-QALY (quality adjusted life year) threshold of $£ 20000$ for commissioning health interventions, suggesting a cost-effective approach.

Conclusion Our Results demonstrate that the liver and cardiometabolic health of patients with NAFLD managed through a multidisciplinary approach show significant improvements. Patients with poorly controlled T2DM had the greatest improvement in $\mathrm{HbA} 1 \mathrm{c}$ of a magnitude known to reduce complications, which may potentially confer good benefit to patients in slowing NAFLD progression. Furthermore, our economic analysis suggest that this approach may be costeffective.

\section{PWE-076 IMPACT OF CIRRHOSIS SEVERITY ON SURVIVAL IN HEPATOCELLULAR CARCINOMA}

Robert Driver*, David Chizhande, Rebecca Jones, lan Rowe. Leeds Teaching Hospitals, Leeds, UK

\subsection{6/gutjnl-2018-BSGAbstracts.218}

Introduction Treatment allocation and overall survival in hepatocellular carcinoma (HCC) is determined by both cancer characteristics and the severity of underlying liver disease. Treatments with the greatest chance of providing cure are often contraindicated by advanced cirrhosis. Routine healthcare data may be used to establish survival following different treatment modalities, but in the absence of biochemistry laboratory Results, few data exist to determine cirrhosis stage at HCC presentation in population-based studies. We present the Results of a pilot study to determine liver disease severity using routinely collected diagnosis and treatment codes related to cirrhosis in hospital episodes at a regional hepatobiliary cancer centre in the UK.

Methods All patients registered within three local Leeds clinical commission groups (CCGs) with a new diagnosis of HCC over a two year period (January 2013 to December 2014) were identified. Using hospital episode codes related to varices and ascites, an algorithm was developed to determine cirrhosis severity as defined by the Baveno stage. Patients were stratified according to decompensation status: compensated cirrhosis by Baveno 1 and 2 and decompensated cirrhosis by Baveno 3 and 4. This staging was validated by comparison with clinical records. Data related to demographics, liver disease aetiology and treatment allocation were collected, along with laboratory data to compare with MELD and Child Pugh (CP) scores. Kaplan-Meier survival analysis was used to compare outcomes by liver disease severity.

Results Among 78 patients with a new diagnosis of HCC (median age 69 years, 61 (78\%) male), 54 patients (69\%) had evidence of cirrhosis at presentation. The most frequent underlying disease aetiologies were hepatitis C (26\%) and alcohol-related liver disease (24\%). Patients with compensated cirrhosis had a median survival of 22.9 months and those with decompensated cirrhosis it was 2.6 months $(p=0.014)$. The decompensated group had a median CP score of 9 and MELD of 13, compared with a median CP score of 5 and MELD of 10 in the compensated group. The Baveno algorithm correctly determined the Baveno score in 53/54 (98\%) patients with cirrhosis.

Conclusions This pilot study demonstrates the successful use of an algorithm to determine Baveno stage using diagnosis and procedure codes from inpatient hospital episodes. This scoring system correlates with other validated prognostic scores in cirrhosis. In patients with HCC, the severity of the underlying liver disease must be assessed when considering outcomes for these individuals. It is expected that this algorithm will be used by the HCC-UK/National Cancer Registration and Analysis Service partnership in forthcoming population-based studies of HCC outcomes in England.

\section{PWE-077 SCREENING FOR NON-ALCOHOLIC FATTY LIVER DISEASE IN PRIMARY CARE USING SIMPLE FIBROSIS MARKERS}

Kate Earp*, Amer Al-Joudeh, Hannah Delaney. Sheffield Teaching Hospitals NHS Foundation Trust, Sheffield, UK

\subsection{6/gutjnl-2018-BSGAbstracts.219}

Introduction Non-alcoholic fatty liver disease (NAFLD) is a significant public health concern. Rates are increasing due to increasing levels of obesity. Early identification of patients in primary could prevent progression to end stage liver failure. The aim of this project is to pilot introduction of a screen for NAFLD and liver fibrosis into the existing NHS Health Check. Simple fibrosis scores have been extensively evaluated in a secondary care setting, however their utility in primary care has not been established.

Methods Five GP practices took part in the pilot. The NHS Health Check is offered by GPs to any patient aged 40-74 years who is not already on a disease register. Patients who attended for this were screened to determine if they met the 
inclusion criteria. Patients were included if they had an increased BMI ( $\geq 30$ or $\geq 27.5$ in high risk ethnicities) or waist circumference $(>94 \mathrm{~cm}$ men or $>90 \mathrm{~cm}$ men high risk ethnicities and $>80 \mathrm{~cm}$ women) and low alcohol intake ( $<28$ units/ week males or $<21$ units/week females). AST/ALT ratio, Fibrosis-4-score (FIB-4) and NAFLD score were calculated for each patient. Those with an increased fibrosis score were reviewed in outpatients and had further investigations; non-invasive liver screen, liver ultrasound scan and liver elastography.

Results Out of the 82 included patients, 64 (78\%) had at least one increased fibrosis score. Twenty-six patients with raised fibrosis score/s were reviewed in outpatient clinic and were offered further investigations. Of 15 patients who had an US scan $10(66 \%)$ had fatty liver and the rest were normal. Twenty-one patients had liver elastography and 1 was found to have possible fibrosis with a fibrosis score of $8.1 \mathrm{kPa}$. Subsequent liver histology showed fibrosis, however it was thought to be drug induced.

Conclusions Informal feedback from the staff performing the NHS Health Check indicated that incorporating the liver screen was not burdensome. However, based on these Results simple fibrosis markers are not a useful screening test for use in primary care, as the high false positive rate Resulted in too many unnecessary referrals to secondary care to exclude fibrosis. It remains unclear how patients with liver fibrosis can be easily identified in primary care.

\section{PWE-078 SPONTANEOUS BACTERIAL PERITONITIS PROPHYLAXIS: ARE WE FOLLOWING GUIDELINES?}

Sun Mi Ha*, Hannah Al-Hasani, Laura Crisan, Vicky Jeyaprakash, Nabil Siddique. East Kent Hospitals NHS Foundation Trust, UK

\subsection{6/gutjnl-2018-BSGAbstracts.220}

Introduction Spontaneous bacterial peritonitis (SBP) is the most common serious infection in patients with cirrhosis, occurring in $25 \%$ of those who develop ascites. It is associated with significant morbidity and mortality rates of $20 \%-40 \%$. ${ }^{1}$ British Society of Gastroenterology (BSG) and National Institute of Clinical Excellence (NICE) guidelines recommend long-term prophylaxis (LTP) with Ciprofloxacin or Norfloxacin in patients with cirrhosis who have low ascitic fluid protein concentration $(<15 \mathrm{~g} / \mathrm{L})$ with or without prior episode of SBP (primary LTP) or who have had an episode of spontaneous bacterial peritonitis (secondary LTP). ${ }^{1} 2$

Methods We carried out a retrospective observational study using our electronic system for admissions with a diagnosis of ascites and cirrhosis across the East Kent Hospitals NHS Foundation Trust from April 2014 to April 2017. Ascitic fluid analysis Results were reviewed against discharge summaries to audit whether LTP was started according to national guidelines.

Results 337 cases of ascites with cirrhosis were identified (93 female: 244 male) with a median age of 58 (range 30-92 years). 61 out of 337 cases had a current or previous diagnosis of SBP. 5 out of 61 died during their admission. 10 out of 61 were discharged on secondary LTP and 46 patients were discharged without LTP. 11 out of 337 cases had low ascitic fluid protein with no current or previous episodes of SBP. None of these patients were discharged with primary LTP.
Conclusions East Kent Trusts followed national guidelines in starting secondary LTP for SBP in 18\% (10 out of possible 56 ) of cases and $0 \%$ of cases requiring primary LTP from April 2014 to April 2017. This low adherence rate may reflect lack of clinician awareness of guidelines for prescribing LTP for SBP in patients with ascites. There may also be a relation to local microbiology guidelines not following BSG or NICE guidelines on initiation of primary or secondary LTP for SBP. This study serves as a reminder to clinicians to carefully consider LTP in patients with ascites secondary to cirrhosis on each admission. We also recommend that trusts review local microbiology guidelines to ensure it adheres to national guidelines.

\section{REFERENCES}

1. Cirrhosis in over 16s: assessment and management. NICE Guidelines, July 2016.

2. Moore KP, Aithal GP. Guidelines on the management of ascites in cirrhosis. Gut. 2006;55(Suppl 6):vi1-vi12. doi:10.1136/gut.2006.099580

\section{PWE-079 DIAGNOSTIC UTILITY OF AUTOIMMUNE SEROLOGY PROFILING}

${ }^{1,2}$ Debashis Haldar* ${ }^{3}$ Tim Plant, ${ }^{4}$ Olivia Serevina, ${ }^{4}$ Kenneth Chung, ${ }^{2}$ Kashif Qamar,
${ }^{1,2}$ Bridget Gunson, ${ }^{2}$ Andrew Holt, ${ }^{1,2}$ Alex Richter, ${ }^{1,2}$ Gideon Hirschfield. ${ }^{1}$ Institute of
Immunology and Immunotherapy, University of Birmingham, Birmingham, UK; ${ }^{2}$ University
Hospitals Birmingham NHSFT, Birmingham, UK; ${ }^{3}$ Department of Clinical Immunology,
University of Birmingham, Birmingham, UK; ${ }^{4}$ College of Medical and Dental Sciences,
University of Birmingham, Birmingham, UK

10.1136/gutjnl-2018-BSGAbstracts.221

Introduction Immunoserologic investigation is of clinical use in the discrimination of liver disease of autoimmune aetiology from alternate causes. We sought to investigate the diagnostic utility of immune serology in patients investigated for liver disease.

Method We analysed the immunoprofile of patients investigated for liver disease at a tertiary centre between 2001 and 2017. We compared Results for patients clinically coded with a diagnosis of autoimmune hepatitis (AIH), primary biliary cholangitis (PBC), or primary sclerosing cholangitis (PSC) against those without, amongst patients investigated by our liver services. Overlap features $(\mathrm{o} / \mathrm{l})$ were included if clinically documented.

Results We evaluated Results from 2874 patients. Patients with AIH ( $n=556$; incl. o/l (19.3\%)) were predominantly female (3 F: $1 \mathrm{M})$, with a bimodal age distribution at presentation $(\approx 18$, 62 years). Antinuclear (ANA) and smooth muscle antibodies (SMA) were both sensitive $(92.3 \%, 86.6 \%$ respectively) for a diagnosis of $\mathrm{AIH}$, but poorly specific (23.6\%, 27.2\%). Amongst patients with $\mathrm{T}_{1} \mathrm{AIH}, 39$ patients had antibodies to soluble liver antigen (SLA). Of these, 36 (92\%) had concordance to Ro-52 antibodies. $2.3 \%$ of patients with $\mathrm{AIH}$ had $\mathrm{T}_{2} \mathrm{AIH}$, defined by antibodies to liver-kidney microsome-1 (LKM); of the 13 patients, 4 had SMA and 8 have ANA reactivity. Patients with $\mathrm{PBC}(\mathrm{n}=527$; incl. o/l $(18.3 \%))$ were primarily female (9 F: $1 \mathrm{M}$ ), and in their 6th decade (mean $=56$ years). Anti-mitochondrial antibody (AMA) testing was both sensitive (70.6\%) and specific (91.2\%). In patients who were AMA negative at testing, anti-glycoprotein 210 (gp210) (sens 40.7\%, spec 95\%) and anti-SP100 (sens 40.3\%, spec 96.3\%) proved useful diagnostic adjuncts. Patients with PSC $(n=291$; incl. o/l (10.1\%)) were predominantly male (3 